\title{
Bibliotecas y sociedad: el paradigma social de la biblioteca pública
}

\author{
Felipe Meneses Tello *
}

\footnotetext{
Artículo recibido:

9 de mayo de 2013.

Artículo aceptado:

25 de junio de 2013.
}

\begin{abstract}
Resumen
El autor analiza aspectos teóricos en torno a la relación "bibliotecas y sociedad". En concreto discute cuatro ideas fundamentales relacionadas con el paradigma sociológico de la biblioteca pública: 1) la biblioteca pública como institución social; 2) los postulados sociales de la biblioteca pública; 3) el ethos social de la biblioteca pública y 4) la biblioteca pública como constructora social de la comunidad. Así, se concluye que esta institución bibliotecaria contribuye a construir una mejor ciudadanía, es decir, una ciudadanía educada e informada con la finalidad de hacer funcionar mejor el Estado democrático.
\end{abstract}

Palabras clave: Biblioteca pública; Sociedad; Institución social; Paradigma social.

* Facultad de Filosofía y Letras de la UNAM, México. fmeneses@unam.mx

INVESTIGACIÓN BIBLIOTECOLÓGICA, Vol. 27, Núm.61, septiembre/diciembre, 2013, México, ISSN: 0187-358X. pp. 157-173 


\section{Abstract}

\section{Libraries and society: the social paradigm of the public library}

Felipe Meneses-Tello

The author analyzes theoretical aspects associated with the so-called "library-society" relationship paradigm. The scope to the discussion includes: 1) the public library as a social institution; 2) the social principles of the public library; 3 ) the social ethos of the public library, and 4) the public library as builder of the community. The paper concludes that this library institution contributes to building better citizenry, i.e., a more educated, better informed citizen who can participate in society while enhancing the operation of the democratic state.

Keywords: Public library; Society; Social institution; Social paradigm.

\section{INTRODUCCIÓN: LOS ENFOQUES SOCIALES DE LA BIBLIOTECA PÚBLICA}

Si el libro, como todo tipo de documento, es histórica y culturalmente un Oinvento social del ser humano, la biblioteca es también una "invención social” (Shera, 1976: 13), es una "creación social” (Shera, 1970: 52). Es, en todo caso, una obra social que ha ideado la humanidad a lo largo de los siglos para servirse de ella. Así, podemos concordar con dos planteamientos generales: 1) "La biblioteca ha sido desde sus comienzos, hasta donde conocemos, un instrumento social" (Shera, 1972: 314) y 2) "La biblioteca es un fenómeno social en el mundo social con sus funciones sociales" (Rajam, 1997: 203). Dimensión sociológica de la que es factible desglosar el prototipo social de la entidad bibliotecaria en cuestión.

La base teórica esencial del paradigma social de la biblioteca pública es, como de todos los tipos de bibliotecas, la relación que existe entre "bibliotecas y sociedad" (Bostwick, 1920; Shera, 1976; Chubarian, 1976; Sharma, 1987; Traniello, 2005; Sridevi y Vyas, 2005; Baker y Evans, 2011). La columna teórica fundamental que sostiene este modelo es el estudio y análisis de esta naturaleza de biblioteca como "institución social" (Ballard, 1936; Martin, 1937; Murison, 1955; Rath y Rath, 1993; Kinnell y Sturges, 1996; Moncada, 
2008; Hansson, 2010). Esta idea contrasta, desde una visión amplia, con quien observa a las bibliotecas como una "agencia social" (Reith, 1984) y, desde un punto de vista ajustado a nuestro objeto de estudio, con quien discute este concepto (Shera, 1949) y ha estado de acuerdo con el mismo (Shera, 1970). En este marco reflexivo de particular relevancia es como se ha logrado argumentar que este centro de acervos y servicios tiene primordialmente un determinado conjunto de funciones sociales a desempeñar que a menudo se plantea como una múltiple "función social".

Los estudios y análisis sociológicos en torno a la bibliotecología tienen poco tiempo si consideramos que en los Estados Unidos "la biblioteca solamente ha sido reconocida como entidad sociológica durante la última mitad del siglo" (Shera, 1972: 316) y en otros países, como los latinoamericanos, esos estudios tienen menos tiempo al privilegiar principalmente la enseñanza y la investigación sobre aspectos técnico-administrativos. No obstante, en estos cuadrantes epistemológicos las bibliotecas públicas han sido uno de los principales objetos de estudio de la investigación con perfil sociológico. Una de las mejores interpretaciones sociológicas de la biblioteca pública, en términos de conceptos aceptados por la ciencia social (Egan, 1955: 15), data de hace más de 70 años. En efecto, en esa apreciación el autor trató temas fundamentales como la naturaleza social, las funciones sociales, los orígenes sociales, el cambio social y los valores sociales de la biblioteca pública (Martin, 1937).

Resulta relevante, desde otro ángulo, reconocer que el artículo "bibliotecas" esté registrado en la International encyclopedia of the social sciences (Shera, 1972: 301); y que al concepto de "biblioteca pública", como expresión bibliotecológica, se le considere oportuno incluirlo en un Diccionario de Sociología, en el que se define a esta institución como la que es "costeada con fondos públicos y que se halla gratuitamente al servicio de toda la población" (Fairchild, 1987: 24). Si bien estos términos, el simple y el compuesto, no son propiamente conceptos sociológicos, estas referencias y el presente paradigma social nos orientan, asimismo, para afirmar que las palabras "biblioteca(s)" y "biblioteca(s) pública(s)" son susceptibles de valoraciones de tal naturaleza. El estudio sociológico de Lloyd V. Ballard (1936: 227) en torno a esta institución bibliotecaria en su obra Social institutions es también un ejemplo claro al respecto.

\section{LA BIBLIOTECA PÚBLICA COMO INSTITUCIÓN SOCIAL}

Diferenciar una biblioteca pública como institución o agencia con perspectiva sociológica tiene importancia porque ambos conceptos denotan nivel en 
el marco de la estructura social. Quienes la aprecian como institución social la perciben como símbolo cultural que existe para conservar y transmitir la herencia cultural de la sociedad (Shera, 1976: 49). Quienes la distinguen como una agencia social la consideran como una pieza clave para hacer funcionar una gran variedad de instituciones políticas, educativas, científicas, económicas, religiosas, gubernamentales, etcétera, al servicio de la sociedad (Shera, 1970: 60; Reith, 1984: 6). Shera nos ilustra para entender esta disparidad:

La biblioteca pública ha sido considerada como una agencia social en lugar [...] de una institución social. La distinción es fundamental para completar la comprensión de la relación entre la biblioteca y su entorno social. Considerando que la institución social es primaria y básica, la agencia social es secundaria y derivada. La familia y el Estado son las instituciones; la escuela, la biblioteca y el museo son las agencias. Unas las determinan el modelo de la sociedad, y las otras son determinadas por ese modelo. La agencia es el instrumento de la institución, y por medio de ella la institución ejerce gran parte de su control social. Así, la distinción es más que una cuestión de grado, pues implica un flujo de poder y autoridad (1949: v).

Unos años antes el sociólogo Llody V. Ballard en su obra Social institutions había reflexionado en torno a este asunto en el capítulo XII, intitulado "The public library". En ese apartado el autor argumenta que la concepción de la biblioteca pública como una institución complementaria a la escuela es lo que originó la idea que denota subordinación puesto que a este centro bibliotecario se le confirió el estatus de una agencia complementaria al centro escolar. Idea con la que no concuerda porque: "La biblioteca pública, como resultado, se ha estado convirtiendo rápidamente en una institución social por derecho propio" (Ballard, 1936: 233). Con el paso del tiempo esta visión fue fortaleciéndose a tal grado que es común leer en la literatura especializada puntos de vista como éste, que asevera:

Entre las instituciones sociales que deben contribuir al desarrollo de las manifestaciones culturales de la gran mayoría de la población y proporcionar mejores condiciones para el tratamiento de sus necesidades básicas de supervivencia, y renovar los lazos de la ciudadanía común, [está] la biblioteca, en particular, la biblioteca pública (Cysne, 1993: 16).

Así, pensamos que la noción de la biblioteca pública como institución social se vincula con el concepto de "sistema"; mientras que como agencia so- 
cial se relaciona con la idea de "parte del sistema". El rango social entre una expresión y otra es claro en este sentido. Empero, aunque ambos puntos de vista tienen lógica, nos inclinamos por valorar a este centro bibliotecario -en virtud del estatus que éste se ha forjado alrededor del mundo y del consenso reflexivo que se ha alcanzado en torno a esta concepción entre los bibliotecólogos estudiosos del tema- como una institución social. Es decir, concebimos esta biblioteca como un sistema bibliotecario público equiparable, por ejemplo, al sistema educativo-básico-público. Sistemas que en la práctica, como se ve a diario, son complementarios para la sociedad pero autónomos en la esfera de la estructura de la Administración pública del Estado en la que se inserta el Sistema Bibliotecario Nacional.

Se asevera que las bibliotecas públicas estadounidenses fueron las primeras en ser definidas como instituciones sociales, resultado del movimiento democrático que caracterizó al siglo XIX (Hansson, 2010: 5). A partir de entonces, la idea inherente a la biblioteca como una institución social se ha hecho tan potente que es, sin duda, uno de los principios sustanciales que orienta el trabajo bibliotecario de hoy en día. Motivo por lo que esta noción se ha convertido, desde una visión de razón teórica, en uno de los supuestos universales de la bibliotecología y, desde una arista de sentido práctico, en un tópico general de la praxis bibliotecaria. De tal modo que la premisa referente a la biblioteca pública como una institución social presenta un valioso perfil sociológico, el cual se puede sintetizar como sigue: 1) ideológicamente adoptó la estructura de una organización social que nació con la biblioteca moderna no sólo como un recurso, sino incluso como una solución normativa a la selección, recolección, organización y ejecución para el amplio uso social de la información; 2) aseguró la supervivencia de una sociedad por haberse convertido en la principal agencia para que la información sea fácilmente accesible a los miembros de la sociedad, y 3) es el legado cultural más significativo para la sociedad (Miksa, 1996). Acorde con este contorno, se infiere: "El reconocimiento de la biblioteca pública como una institución social de usos múltiples es ahora mundial, como mucho se aprecia en la interpretación flexible de sus objetivos en todo el mundo" (Murison, 1988: 85).

En efecto, desde una perspectiva general, la biblioteca como institución social es un tópico de estudio sociológico que versa acerca del servicio de biblioteca (Rajam, 1997: 202); desde una vertiente específica, es una de la ideas básicas en el plano bibliotecario, y la base de lo que se ha convertido en uno de los supuestos comunes del campo de la biblioteca moderna y de sí misma (Miksa, 1996). Este razonamiento, vinculado con el paradigma en cuestión, ha permitido configurar teóricamente el nexo "bibliotecas y sociedad" en una articulación disciplinaria entre "biblioteconomía y sociología" (Rath y 
Rath, 1993; Almeida Júnior, 1997) y entre "bibliotecología y sociología" o, de manera más explicita, en distinguir una gran variedad de fenómenos clave inherentes a la investigación sociológica de la biblioteca en general y de la biblioteca pública en particular, cultivando así una esfera cognitiva referente a la especialidad conocida como bibliotecología social.

\section{LoS POSTULAdOS SOCIALES DE LA BIBLIOTECA PÚBLICA}

En la literatura especializada se identifican algunos postulados que sirven de fundamento en relación con la base-columna teórica de este paradigma. Uno de ellos es el que admite que la biblioteca pública tiene una notable "responsabilidad social" en los diversos cuadrantes de la sociedad (Henri y Lee, 2007; Baker y Evans, 2011); otro es el referente al "compromiso social" que su personal bibliotecario contrae con la comunidad a la que atiende (Toledo de Araújo, 1985); y uno más es el inherente a la percepción que vislumbra a estos centros bibliotecarios públicos como catalizadores proactivos de "cambio social" (Pungitore, 1989; Haywood, 1997; Yilmaz, 2002; Mehra y Srinivasan, 2007). Frente a los desafíos que engloban una seria problemática social, estos principios apuntan hacia la necesidad de adoptar una "conciencia social" por parte de quienes hacen funcionar las bibliotecas públicas.

Consecuentemente, en torno a este paradigma gira la serie de paradigmas sociológicos de la biblioteca destinada para todos los grupos que conforman la sociedad. Paradigmas tales como el educativo, el cultural, el popular, el comunitario, el indígena, el multicultural y otros quizás aún indistinguibles. Perspectiva caleidoscópica que configura y engarza la "misión social" atribuida a las bibliotecas públicas de acuerdo con una evolución histórica caracterizada por una serie de claroscuros (Fernández, 2006). Del cumplimiento de su misión pensamos que depende el "impacto social" de éstas porque:

En el proceso de evolución y adaptación a determinados lugares, las bibliotecas públicas se han convertido en parte de la estructura de la sociedad civil. Son una fuente de apoyo para los diferentes tipos de actividades. Las bibliotecas pueden ser una piedra angular para ayudar a sostener la vida de una localidad. Son utilizadas por individuos y grupos que llevan a cabo la actividad del día a día en el que mantiene a la sociedad en marcha. Esto es lo que hemos denominado el "impacto social" de la biblioteca (Greenhalgh, Worpole y Landry, 1995: 89).

Asimismo, nos identificamos con principios generales que nos orientan y revelan el valor histórico de donde emerge y se desarrolla la misión social 
de estos recintos: las bibliotecas son creadas por la sociedad y las bibliotecas son conservadas por la sociedad (Thompson, 1977: 204-205). Puntos de vista análogos aseveran: "La biblioteca es producto de la sociedad y no la base de la sociedad" (Reith, 1984: 7); "La biblioteca es una agencia creada por la sociedad para documentar las variaciones encontradas en los entornos natural y social" (Reith, 1984: 8). Nociones que nos ayudan a generar un fundamento social con un fin y nivel superior: las bibliotecas en general y las bibliotecas públicas en particular existen para el uso de los individuos y de la sociedad. Con base en estos aforismos, podemos coincidir con la idea: "las bibliotecas son indispensables para la sociedad" (Sharma, 1987: 1-9). Y lo son porque han sido creadas esencialmente para satisfacer las necesidades sociales (Shera, 1976: 42) de todos los miembros, grupos e instituciones que se interrelacionan en el marco de la estructura social. En suma, como se reconoce: "Las bibliotecas nacieron del ideal de mejorar la sociedad, un propósito o meta generalmente aceptado" (Wheeler y Goldhor, 1970: 16). Punto de vista que continúa proyectándose con nuevos enfoques y mayores bríos para continuar desarrollando la sociedad.

Observamos así que el paradigma social de la biblioteca pública tiene estrecha relación no solamente con puntos de vista sociológicos, sino también filosóficos, históricos, políticos, antropológicos y otros. Por lo tanto, el fenómeno social de este servicio de biblioteca es complejo por la diversidad de aristas que pueden ser y han sido analizadas. De modo que la serie de premisas sociales de este tipo de biblioteca apunta a entender la necesidad de construir una mejor sociedad. Una sociedad que se distinga por ofrecer oportunidades "para ser mejores ciudadanos" (Wheeler y Goldhor, 1970: 25). El acceso a la lectura para todos, mediante el servicio público de biblioteca, es una forma cultural para lograr esa mejoría social.

La "función social" de la biblioteca pública ha sido y es uno de los argumentos típicos que gira en torno a este tipo de servicio bibliotecario, disponible idealmente para todos, tal como se proclama en las diversas versiones (1949, 1972, 1994) del Manifiesto para la Biblioteca Pública. El "significado social" de estas bibliotecas se ha tratado de explicar, en efecto, a través del reconocimiento de esta gran función. Así, por ejemplo, se asevera que "la función social de las bibliotecas públicas es apoyar los valores fundamentales de la democracia constitucional: la libertad y la igualdad" (Savenije, 2011: 204). Con base en este pensamiento, la importancia social de esta institución de lectura pública se extiende, según afirma este mismo autor, a los dominios de la democracia, la educación, la cultura, la economía y el tiempo libre (2011: 204). Entorno en el que se distingue, explícita o implícitamente, la misión social que debe cumplir este tipo de centros bibliotecarios, la cual es sumamente 
amplia puesto que engloba, en el marco de los fundamentos de nuestra disciplina, la satisfacción de las necesidades de educación, recreación, información y cultura de sus comunidades (Rubi, 2010: 173).

\section{El ethos SOCIAL DE LA BIBLIOTECA PÚBLICA}

El criterio de uso público, de manera individual o colectiva, es lo que ha determinado a través del tiempo el carácter de orden social de las bibliotecas públicas. A consecuencia de las necesidades sociales de información, educación, recreación y culturales en general que presentan los diversos individuos y grupos, se hace visible el uso de las colecciones y de los servicios, fenómeno que produce el vínculo social entre las bibliotecas y la sociedad. Con base en esto, "la interacción social es la esencia del servicio de biblioteca" (Rajam, 1997: 202), es decir, son las diversas relaciones sociales (persona-persona, persona-grupo, persona-institución, grupo-grupo y grupo-institución) que se suscitan entre lectores, usuarios y bibliotecarios lo que dinamiza a la biblioteca pública como valiosa institución social de bienes y servicios públicos. De tal modo que como institución de servicios esta "[...] biblioteca sirve a todas las instituciones de la sociedad y así puede ser reconocida como una fuerza esencial de integración” (Reith, 1984: 6), en este caso social, para coadyuvar a lograr mejores niveles de vida, especialmente de personas y grupos en condiciones de vulnerabilidad.

Sin duda, este tipo de biblioteca resulta intrínsecamente valioso para los individuos y para los grupos sociales. Las bibliotecas públicas se convierten así en el centro intelectual de la comunidad, permitiendo ampliar y profundizar en el contenido de sus colecciones bibliográficas; enriqueciendo tanto la vida colectiva como la personalidad individual a través de los servicios bibliotecarios dirigidos a la generalidad (individuos y grupos) de la población. Propicia de este modo el intercambio de las experiencias que transmiten unos (autores) a otros (lectores y usuarios). Dicho de otra manera: "Las bibliotecas, como laboratorios, son talleres que operan bajo la dirección de un personal capacitado. En ellas, el individuo puede aprender a resolver el problema de su particular situación social recurriendo a la experiencia acumulada de otros" (Ballard, 1936: 231-232).

En este plano reflexivo, es una institución social de mayor bien, de máxima utilidad y de sumo bienestar puesto que sirve, si no a todos, como sugiere la teoría con visiones idealistas, sí al mayor número posible de personas. Este es el ethos esencial de la biblioteca que nos ocupa. Así que la utilidad social de la biblioteca pública es un principio ético-teleológico que apunta a determinar 
la concepción de lo social con base en la finalidad que persigue esta entidad de servicio público en las diversas esferas de la sociedad. En otras palabras: "La importancia de las bibliotecas públicas se puede medir por el efecto para el bien que tienen en la sociedad" (Murison, 1988: 2). En el plano de la sociología, es una institución que forma parte de la vida en sociedad de hombres y mujeres; desde la óptica de la ciencia política, puede observarse como un servicio público que permite la libertad y la igualdad de acceso a la información para ayudar a construir una sociedad democrática; en la esfera de la bibliotecología es la institución social que forma parte de la cultura bibliográfica y bibliotecaria al servicio de la comunidad. Con estas tres apreciaciones podemos aseverar que estas bibliotecas tiene un peculiar valor social, político y documental.

El cumplimento de su responsabilidad-compromiso social, por ende, debe estar orientado en virtud de su reconocida misión ante la sociedad. Su responsabilidad social es, en consecuencia, ante los diversos individuos y grupos que debe atender y en concordancia con las necesidades sociales de información que unos y otros presentan en torno a diversos problemas y eventualidades. Es socialmente responsable así ante las presentes y futuras generaciones de ciudadanos. El compromiso social es por parte del recurso humano que la hace funcionar de manera debida. De modo que esta institución bibliotecaria es valorada y apreciada por el público al que asiste, por ende, es una "institución aprobada" por la sociedad en tanto es una "institución operativa" (Ballard, 1936: 12) que obra para el objeto de estudio de la sociología, esto es, la sociedad.

Con base en estos razonamientos concordamos con la idea: "Si la biblioteca pública no responde a las necesidades sociales, no puede tener éxito en responder a las necesidades de los individuos" (McCabe, 2001: 121), pues el bienestar de los grupos no se puede separar de la prosperidad de los individuos y viceversa. En todo caso, la satisfacción social que puede lograr esta institución entre los grupos que constituyen la comunidad a la que sirve está en estrecha relación con la satisfacción de cada una de las personas que los conforman.

Desde este ángulo, sus acervos, recursos y servicios, según podemos distinguir entre el texto y el contexto, son socialmente útiles. La importancia de la biblioteca pública radica, por ende, no en la cantidad acumulada de sus colecciones sino en la calidad de éstas y de sus servicios disponibles para ser utilizados por mujeres y hombres, niñas y niños; esto es, el peso específico de esta biblioteca reside en el interés de apoyar el pleno desarrollo de la sociedad, es decir: "El papel de las bibliotecas en la sociedad se manifiesta justamente en el proceso de prestación de servicio al lector y en la utilización 
de los fondos por parte del pueblo" (Chubarian, 1976: 63). En congruencia con este punto de vista:

Debe reiterarse que la biblioteca como institución social tiene que ver con la gente, sobre todo con la gente, y luego con sus operaciones; para el pueblo es el objetivo al que se dirige el servicio y para el que existe. Por lo tanto, cualquiera que sea la biblioteca o haga o deje de hacer tiene que confrontarse con las necesidades de la comunidad (Murison, 1988: 225).

El ethos social de la biblioteca pública está vinculado estrechamente, según observamos, con tres relaciones sustanciales: 1) "biblioteca-comunidad", 2) "biblioteca-pueblo" y 3) "biblioteca-sociedad". Estos nexos los encontramos explícita e implícitamente expresados en la primera versión del Manifiesto de la Unesco sobre la Biblioteca Pública (1949) al considerar a ésta como: a) fuerza vital para la comunidad, b) universidad del pueblo y, en consecuencia, c) fuerza viva de la sociedad. Estas ideas implican que esta institución de servicio al público "es una institución al servicio del pueblo", que "está dirigida a los distintos sectores de la comunidad" (Casa, 1974: 41-42). Por lo tanto, es un organismo de servicio público que "contribuye a la creación y el mantenimiento de una sociedad bien informada y democrática" (The Public Library Service, 2001: 8). Consecuentemente, el objetivo primordial de la biblioteca pública es servir a mujeres y hombres de todas las edades, creencias y clases sociales que conforman la comunidad, el pueblo o la sociedad. Esta filosofía de servicio público no observa al usuario en abstracto sino al usuario como ciudadano, como ente social con derechos y responsabilidades en esos tres ámbitos que estructuran la vida colectiva. Desde esta perspectiva, se sugiere apreciar y practicar el servicio de biblioteca pública con espíritu de aprovechamiento común, de bien común público. En este nivel de miras, aseveramos que esta institución funciona con bienes comunes valiosos para contribuir a la vida en común.

En concordancia con estas ideas, el ethos en cuestión lo podemos concebir como el punto de partida y de llegada; como la tendencia y el valor, y como el carácter y la identidad de la biblioteca destinada a servir a todos los grupos que conforman la compleja entidad entre comunidad-pueblo-sociedad. Se trata de una entidad sociológica que se entrelaza. En otras palabras, el ethos social de este tipo de biblioteca nos orienta para reconocer que se trata de una institución que procura hacer el bien, según el enfoque, a la comunidad, al pueblo o a la sociedad. De modo que la palabra ethos, en el contexto de la biblioteca pública, está relacionada con la ética social que se practica o se debe practicar en la esfera de la ética bibliotecaria pública. Concretémonos al nexo teórico biblioteca-comunidad. 


\section{LA BIBLIOTECA PÚBLICA}

COMO CONSTRUCTORA SOCIAL DE LA COMUNIDAD

Pensar que "la labor de la biblioteca pública está dirigida a los distintos sectores de la comunidad" (Casa, 1974: 42) ha implicado reconocer desde hace tiempo el papel integral que debe desempeñar esta biblioteca al interior de la comunidad a través de los servicios que ofrece a los diferentes grupos sociales, especialmente a los grupos vulnerables. Ciertamente, la preocupación por éstos no es nueva. Al reflexionar acerca del trabajo de la biblioteca pública en las comunidades estadounidenses a mediados del siglo XX, Rose señaló la tendencia hacia la comunidad por parte de esta institución de servicio. Según esta autora, los bibliotecarios públicos han mostrado más sensibilidad hacia los grupos más necesitados, a saber: 1) grupos raciales (indígenas, negros, inmigrantes), 2) grupos ocupacionales (obreros, campesinos) y 3 ) grupos discapacitados (ciegos, enfermos) (1954: 39-44). Pero esta sensibilidad, a pesar de estar presente la noción de comunidad, no ha sido semejante en otras latitudes. América Latina es un ejemplo en este sentido.

La literatura latinoamericana en bibliotecología, publicada a lo largo del siglo XX, muestra notoriamente que la relación entre "bibliotecas públicas y comunidades vulnerables" no fue uno de sus primordiales objetos de investigación teórica y empírica, a pesar de que estos grupos sociales estuvieron presentes a la vista prácticamente de toda la sociedad. Esa literatura, saturada de enfoques técnico-administrativos se mantuvo, salvo raras excepciones, aislada del contexto social de las comunidades de usuarios en claros estados de vulnerabilidad social. Esta situación se puede extrapolar para el caso de la educación bibliotecológica impartida durante el siglo recién pasado, pues en los países subdesarrollados de América los planes de estudios de las escuelas pusieron particular énfasis en aquellas asignaturas cuya naturaleza era básicamente técnica. No debe entonces de extrañarnos que en el terreno de la práctica bibliotecaria, en esta región del mundo, habitualmente se haya evidenciado un alto grado de omisión, desinterés e incuria para atender a esos grupos necesitados mediante el servicio de biblioteca pública.

$\mathrm{Si}$ bien es cierto que a mediados del siglo XX, al reflexionar en torno a la misión de la biblioteca pública modelo, se aseveraría que estos centros bibliotecarios deben ser "fuerzas vivas al servicio de la comunidad" (Maurois, 1961: 26), esta idea se redujo a un anhelo difícil de alcanzar en algunos países como los de la región latinoamericana por motivos, entre otros, como los antes expresados. Empero, con el paso del tiempo el pensamiento social, en relación con la biblioteca pública al servicio de la comunidad, ha comenzado a forjarse con mayor empeño. De esta manera, en el siglo XXI se amplía y 
profundiza acerca de los tópicos sociológicos de nuestro objeto de estudio. Esta institución bibliotecaria, como organismo de servicio público, es considerada así un bien social en tanto que es un bien público y un bien de utilidad pública (Traniello, 2005: 47). Los valores de utilidad pública y utilidad social son correlativos en la esfera público-social de este tipo de biblioteca que se caracteriza por ser un servicio público gratuito. Asimismo, la creación y el desarrollo de este espacio social de lectura pública continúa identificándose con el desarrollo de la comunidad, pues el servicio de biblioteca para todos se supedita al mejoramiento local de la población. De tal modo que:

La noción de bien público es esencial para el concepto de una biblioteca pública. Ya en el siglo XVIII, la democracia se consideró necesaria para cumplir con ese bien. Así, las bibliotecas públicas modernas fueron originalmente concebidas como servicio al bien público mediante la promoción de la democracia y la educación civil (D’Angelo, 2006: 11).

La biblioteca pública se ha venido concibiendo, en algunos contextos carentes de recursos, como parte primordial de los programas sociales, cívicos y culturales que apuntan hacia el desarrollo de la comunidad (Batten, 1992: 153). Por esto, es parte esencial de aquellos "planes para lograr una comunidad mejor". Es parte porque ni la biblioteca pública ni la escuela pública básica son capaces de asumir toda la responsabilidad social que demanda el desarrollo comunitario; porque el binomio institucional biblioteca-escuela requiere del apoyo de otras organizaciones. No obstante, reconocemos que el trabajo de la institución bibliotecaria pública en el seno de la comunidad puede influir positivamente en las ideas, las actitudes y la conducta de mujeres y hombres para alcanzar el bienestar comunal. Por esto, lo que se hace a través del servicio de biblioteca pública y cómo se hace, en el entramado de los diversos grupos sociales, adquiere suma importancia. Logra, en concordancia con su misión trazada, la categoría de "biblioteca comunal" (Maurois, 1961: 18), pero sin perder las características políticas, sociales y culturales de gestión de la biblioteca pública.

$\mathrm{Al}$ proporcionar a la gente un lugar común de reunión para la lectura, la recreación y el entretenimiento, las bibliotecas públicas ayudan a generar sentimientos de orgullo local y de sentido general de comunidad (Batten, 1992: 78). La lectura de libros, revistas y periódicos apoya así el reconocimiento y la asimilación de valores sociales y morales en beneficio del trabajo por la comunidad. Desde esta arista, estas bibliotecas tienen la oportunidad de convertirse en epicentros, efectivamente, de bien comunal. La cualidad de biblioteca comunal, principalmente en los medios rurales y suburbios urbanos, cobra 
especial relevancia en virtud de que sus colecciones y servicios representan un recurso común para todos los miembros de la comunidad.

El hecho que las instituciones bibliotecarias de perfil público tengan un reconocido origen social y que satisfagan necesidades sociales de una gran diversidad de grupos que constituyen la sociedad requiere subrayar de qué manera ellas, a través de los años, han venido contribuyendo con sus colecciones y servicios en la construcción social de la comunidad. Así, se contempla que las bibliotecas públicas:

1. Sirven de conducto para acceder a la información con el fin de aprender.

2. Promueven la inclusión social y la equidad.

3. Fomentan la participación cívica.

4. Crean un puente a los recursos y estimulan la participación de la comunidad.

5. Crean la vitalidad económica de la comunidad (Scott, 2011: 197).

Por lo expresado hasta aquí, concordamos en que estas bibliotecas generales ayudan a crear el elemento esencial que requiere la democracia como forma de vida, de gobierno y de Estado: una ciudadanía educada e informada, es decir, una comunidad ilustrada. Esta proyección social la destacó de manera inobjetable el profesor emérito de sociología Lloyd V. Ballard al escribir en la primera mitad del siglo XX:

La biblioteca pública, entonces, se convierte en una unidad educativa coordinada con la escuela pública. En los países organizados en los principios democráticos, es obvio que un electorado inteligente sea esencial para el éxito del gobierno. Una ciudadanía inteligente, sin embargo, sólo se puede asegurar cuando el conocimiento contenido en libros y revistas es universalmente accesible. En general, esta es la función de la biblioteca para facilitar la página impresa en el cumplimiento de su misión (1936: 236).

Función social que este autor matizó al explicar el prisma de funciones técnicas, sociales y culturales que, a su juicio, desempeñan las bibliotecas públicas en el marco de una sociedad democrática, a saber:

1. Conserva la herencia social. En los estantes de la biblioteca se guardan la sabiduría y la experiencia registradas de la humanidad.

2. Distribuye información. La biblioteca pública moderna supone también un papel activo como distribuidor de la información. 
3. Selecciona material educativo. En su selección de libros, la biblioteca pública debe considerar las necesidades y deseos de la comunidad.

4. Guía la lectura. Esta biblioteca ofrece materiales de lectura que la comunidad puede utilizar en el desarrollo y enriquecimiento de su vida colectiva e individual.

5. Desarrolla la homogeneidad social. La biblioteca pública puede ayudar a entender los valores comunes a través de la difusión del conocimiento de éstos entre la población de procedencia o filiación extranjera.

6. Proporciona recreación intelectual. Dado que el uso del tiempo libre es un problema social, la biblioteca pública ofrece oportunidades ilimitadas para el uso creativo del ocio, haciendo posible así la recreación intelectual.

7. Produce herramientas intelectuales. La biblioteca pública, como productora de boletines y catálogos bibliográficos, facilita su funcionamiento haciéndolos accesibles para el público.

8. Proporciona laboratorios para la investigación. Esta biblioteca ayuda al estudiante sugiriéndole interesantes estudios literarios y científicos con significado social; proporciona información a diversos profesionales cuyo trabajo guarda íntima relación con el bienestar y el progreso de las personas.

9. Promueve la interacción social. A través de los peculiares servicios de extensión, la biblioteca pública se ha convertido en centro social e intelectual para aquellos que buscan la recreación y el buen compañerismo entre sí por medio de los acervos y servicios que ofrece (Ballard, 1936: 236-239).

$\mathrm{Al}$ considerar estas funciones de las bibliotecas públicas se clarifica por completo la idea de que la biblioteca es, en efecto, una institución social de contenido profundo y no una morada simple de acervos bibliográficos (Ballard, 1936: 239) al servicio egoísta del usuario como individuo, por ende, aislada o al margen de la comunidad-pueblo-sociedad. Estas instituciones bibliotecarias satisfacen así el dominio de las necesidades sociales mencionadas en rubros anteriores. La satisfacción de éstas permite hacer efectivo en la práctica el espacio de libertad que implica el acceso libre a las colecciones y salas de lectura; y el usufructo gratuito de los acervos, servicios y recursos complementarios que brindan estos centros a la población en general. Desde esta perspectiva, este tipo de bibliotecas deben continuar contribuyendo alrededor del mundo a desarrollar más y mejores capacidades y habilidades entre los miembros de la sociedad civil para el ejercicio de sus acciones ciudadanas a realizar en el interior de sus comunidades. 


\section{Conclusión}

Biblioteca y sociedad en general, y biblioteca pública y sociedad en particular, no pueden ignorarse porque la biblioteca, en términos generales y específicos, es una institución social. Lo es primero porque quienes la hacen funcionar, los bibliotecarios, son seres sociales; segundo porque ellos son un puente, un lazo de unión entre los autores y los usuarios, todos ellos miembros de la sociedad; y tercero porque el obrar bibliotecario afecta a individuos y grupos sociales contribuyendo así al análisis y estudio de conceptos, ideas, temas, corrientes y valores; es decir, esta biblioteca es una constructora social de la comunidad porque con los acervos bibliográficos que contiene y los servicios que ofrece satisface necesidades humanas vitales; porque mediante el acceso a sus acervos y el uso de sus servicios esta institución de información contribuye a construir una mejor ciudadanía, esto es, grupos de ciudadanos capaces de hacer funcionar la maquinaria del Estado democrático. De tal modo que la actividad bibliotecaria pública es una actividad humana social, ciudadana y, por ende, políticamente esencial en beneficio de la sociedad y del Estado.

\section{REFERENCIAS}

Almeida Júnior, O. F. (1997), Sociedade e biblioteconomia, São Paulo: Polis, Associação Paulista de Bibliotecarios.

Ballard, L. V. (1936), “The public library”, en Social Institutions, New York: Appleton-Century.

Baker, D. y Evans, W. (2011), "Libraries, society and social responsibility", en Libraries and society: role, responsibility and future in an age of change, Oxford, United Kingdom: Chandos Publishing, pp. 1-16.

Batten, Thomas R. (1992), Las comunidades y su desarrollo: estudio introductorio con referencia especial a la zona tropical, México: Fondo de Cultura Económica.

Bostwick, A. E. (ed.) (1920), The library and society: Reprints of Papers and Addresses, New York: H. W. Wilson Co.

Casa Tirao, Beatriz (1974), "La biblioteca pública", en Bibliotecas y educación, México: Centro para el Estudio de Medios y Procedimientos Avanzados de la Educación, pp. 41-45.

Chubarian, O. S. (1976), "Biblioteca y sociedad", en Bibliotecología general, La Habana, República de Cuba: Ministerio de Cultura, Editorial Científico-Técnica, pp. 57-122.

Cysne, F. P. (1993), Biblioteconomia: dimensão social e educativa, Fortaleza: EUFC.

D'Angelo, Ed (2006), Barbarians at the gates of the public library: how postmodern consumer capitalism threatens democracy, civil education and the public good, Duluth, Minnesota: Library Juice Press. 
Egan, M. (1955), “The library and social structure”, en Library Quarterly, 25 (1), pp. 15-22.

Fairchild, H. P. (ed.) (1987), Diccionario de sociología, México: Fondo de Cultura Económica.

Fernández Abad, F. J. (2006), "Evolución histórica de la función social de las bibliotecas públicas", en Revista General de Información y Documentación, 16 (2), pp. 93-110.

Greenhalgh, L.; Worpole, K. y Landry, Ch. (1995), Libraries in a world of cultural change, London: UCL Press.

Hansson, J. (2010), Libraries and identity: the role of institutional self-image and identity in the emergence of new types of library, $\mathrm{Ox}$ ford: Chandos Publishing.

Haywood, T. (1997), "Public libraries, real space and social change: some old and some new coincidences", en Alexandria, 9 (3), pp. 201-211.

Henri, J. y Lee, S. (2007), Social responsibility and the role of the public library, Oxford: Chandos Publishing.

Kinell, M. y Sturges, R. P. (eds.) (1996), Continuity and innovation in the public library: the development of a social institution, London: Library Association.

Martin, L. (1937), "The American public library as a social institution”, en Library Quarterly, 7 (4), pp. 546-563.

Maurois, André (1961), La biblioteca pública y su misión, París: Organización de las Naciones Unidas para la Educación, la Ciencia y la Cultura.

McCabe, R. B. (2001), "Library services in a social context", en Civic librarianship: renewing the social mission of the public library, Lanahm, Maryland: The Scarecrow Press, pp. 121-129.

Mehra, B. y Srinivasan, A. (2007), "The library-community convergence framework for community action: libraries as catalysts of social change", en Libri, 57 (3), pp. 123-139.

Miksa, F. (1996), "The cultural legacy of the modern library for the future", en Journal of Education for Library and Information Science, 37 (2), pp. 100-119.

Moncada Patiño, J. D. (2008), La biblioteca pública como institución social, Medellín: Universidad de Antioquia, Escuela Interamericana de Bibliotecología, Centro de Investigaciones en Ciencia de la Información.

Murison, W. J. Z. (1988 [1955]), The public library: its origins, purpose and significance, $3 \mathrm{rd}$, London: Harrap.

Pungitore V. L. (1989), “Changes agencies”, en Public librarianship: an issues-oriented approach, New York: Greenwood Press, pp. 37-41.

Rajam, V. (1997), "Sociology of library service”, en Herald of Library Science, 36 (3-4), pp. 201-204.

Rath, M. y Rath, P. (1993), Sociology of librarianship, Delhi: Pratibha Prakashan. 
Reith, D. (1984), “The library as a social agency”, en A. Robert Rogers y Kathryn McChesney, The library in society, Littleton, Colorado: Libraries Unlimited, pp. 5-32.

Rose, Ernestina (1954), "The library in the community", en The public library in American life, New York: Columbia University Press, pp. 38-47.

Rubi, Richard E. (2010), Foundations of library and information science, New York: Neal-Schuman Publishers.

Savenije, Bas (2011), "Libraries in the information society: cooperation and identity", en Libraries and society: role, responsibility and future in an age of change, Oxford, United Kingdom: Chandos Publishing, pp. 203-218.

Scott, R. (2011), "The role of public libraries in community building", en Public Library Quarterly, 30 (3), pp. 191-227.

Sharma, P. (1987), "Library in society", en Libraries and society, New Delhi: Ess Ess Publications, pp. 1-9.

Shera, J. H. (1949), Foundations of the public library: the origins of the public library movement in New England 1629-1855, Chicago, Illinois: The University of Chicago Press.

(1970), Sociological foundations of librarianship, New York: Asia Publishing House.

- (1972), "Libraries", en International encyclopedia of the social sciences, vol. 7, New York: The Macmillan Company.

(1976), "The library and society", en Introduction to library science: basic elements of library service, Littleton, Colorado: Libraries Unlimited, pp. 42-60.

Sridevi, J. y Vyas, S. (2005), Library and society, New Delhi: Shree Publishers \& Distributors.

The Public Library Service: IFLA/Unesco guidelines for development (2001), ed. for the Section of Public Libraries of the International Federation of Library Associations and Institutions by Philip Gill, München: Saur.

Thompson, J. (1977), A history of the principles of librarianship, London: Clive Bingley.

Toledo de Araújo, Walkíria (1985), "A Biblioteca popular e o compromiso social do bibliotecário", en Revista Escola Biblioteconomia da UFMG, 14 (1), pp. 106-122.

Traniello, P. (2005), Biblioteche e società, Bologna: Il mulino.

Wheeler, Joseph L. y Goldhor, Hernbert (1970), Administración práctica de bibliotecas públicas, México: Fondo de Cultura Económica.

Yilmaz, I. B. (2002), "Social change, industrialisation and public libraries: a theoretical approach", en Aslib Proceedings, 54 (5), pp. 326-334. 